\title{
Case Series: Fetal Pulmonary Vein A-Wave Reversal: An Early Marker of Left-Sided Cardiac Anomalies?
}

\author{
Aldo L. Schenone, MD 1 \\ G. Giugni, MD2 \\ M. H. Schenone, $M D^{3}$ \\ L. Diaz, $\mathrm{MD}^{2}$ \\ A. Bermudez, $M D^{2}$ \\ D. Majdalany, $\mathrm{MD}^{4}$ \\ A. Sosa-Olavarria, MD, $\mathrm{PhD}^{2}$
}

${ }^{1}$ Internal Medicine, Medicine Institute, Cleveland Clinic Foundation, Ohio

Address for correspondence Aldo L. Schenone, MD, Internal Medicine

${ }^{2}$ Centro de Estudios Perinatales, Valencia, Venezuela

${ }^{3}$ Department of Materno-fetal Medicine, University of Residency Program, Cleveland Clinic, 9500 Euclid Avenue, NA 10

Tennessee, Memphis

Cleveland, OH 44195 (e-mail: schenoa@ccf.org).

${ }^{4}$ Clinical Cardiology, Heart and Vascular Institute, Cleveland Clinic, Ohio

Am J Perinatol Rep 2015;5:e60-e66.

\author{
Abstract \\ Keywords \\ - fetal pulmonary vein \\ - congenital cardiac \\ anomaly \\ - congenital heart \\ disease \\ - first-trimester \\ screening \\ - fetal heart disease \\ screening
}

Background Improvements in congenital heart disease (CHD) screening are needed based on the lack of sensitivity of current screening methods and the understanding that the early detection of certain CHDs may improve outcomes. Fetal venous circulation has caught medical attention, and two studies demonstrated that it is feasible to register pulmonary vein flow velocity waveforms (FVWs) during early gestation. Meanwhile, the latter study proposed pulmonary vein A-wave reversal as a marker of cardiac anomaly.

Methods We report a series of six consecutive fetuses with confirmed cardiac anomalies that underwent first-trimester screening, including pulmonary vein FVWs, at our center during 2013. CHD was confirmed by late pregnancy echocardiography, and in three cases fetal autopsies were performed.

Result/Discussion The ductus venosus (DV) and nuchal translucency (NT) predicted $50 \%$ of CHD cases, whereas the combination of markers identified $66.6 \%$ of CHD cases. When adding pulmonary vein assessment, the rate of detection rose to $83.3 \%$. Total five of six cases of CHD had reversal of pulmonary vein A-wave during early pregnancy. The sixth case with $\mathrm{CHD}$ and nonreversal of A-wave was described as right ventricle hypoplasia with type 1 tricuspid atresia and persistent ductus arteriosus.

Conclusion This is the first series reporting pulmonary vein end-diastolic reversal as a CHD screening add-on during early pregnancy. The addition of pulmonary vein FVW assessment to the current CHD screening bundle could increase the rate detection of cardiac anomalies. This pilot study suggests that pulmonary vein end-diastolic flow reversal favors detection of left-sided CHD over the right-sided ones.
Improvements in congenital heart disease (CHD) screening are needed based on lack of sensitivity, ${ }^{1}$ and the understanding that the early detection of certain CHDs may improve outcomes. ${ }^{2}$ In this regard, fetal venous circulation has caught medical attention in recent decades. ${ }^{3-8}$ It is understood that changes in the venous system might reflect variations in heart performance. ${ }^{4}$ For instance, Lenz et al reported pulmonary vein A-wave reversal predominantly among left-sided cardiac anomalies during late pregnancy. In contrast, only approximately 0 to $3.3 \%$ of normal fetuses present with pulmonary vein A-wave reversal. 6,9 received

October 7, 2014 accepted after revision December 23, 2014 published online March 4, 2015
DOI http://dx.doi.org/ $10.1055 / \mathrm{s}-0035-1545670$ ISSN 2157-6998.
Copyright $\odot 2015$ by Thieme Medical Publishers, Inc., 333 Seventh Avenue, New York, NY 10001, USA. Tel: +1(212) 584-4662.
License terms

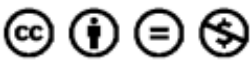




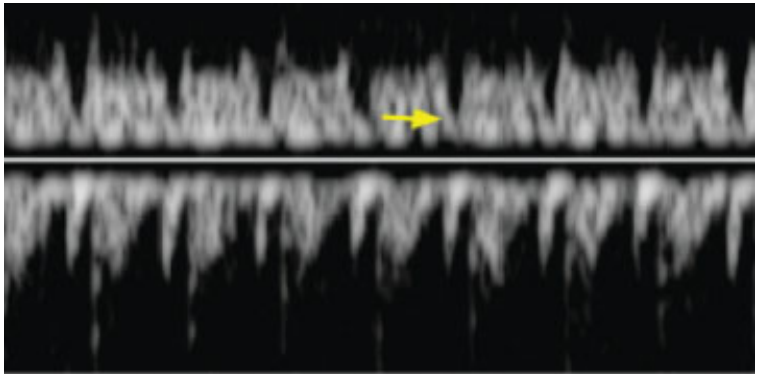

Fig. 1 Normal pulmonary vein flow velocity wave. Arrow point toward forward flow during end diastole (A-wave).

A recent study demonstrated the feasibility to register pulmonary vein flow velocity waveforms (FVWs) during early gestation by X-flow. ${ }^{6} \mathrm{X}$-flow (enhanced-flow) is an ultrasound-based technology that provides a high spatialtemporal resolution by a shorter pulse length when applying a composite pulse emission technique. This translates into better resolution of blood flow dynamics in a low flow state, as well as, the capacity to differentiate between blood flow and surrounding tissue. ${ }^{10}$ Subsequently, Schenone et al confirmed this finding and proposed pulmonary vein A-wave reversal as a first-trimester marker of cardiac anomaly (-Fig. 1) ${ }^{8}$ In response to this hypothesis, we present a case series of consecutive patients with confirmed CHD who had first-trimester pulmonary vein FVWs assessment.

\section{Methods}

We report a series of six consecutive patients with confirmed cardiac anomalies, who underwent first-trimester screening at
“Centro de Estudios Ultrasonograficos Perinatales," Venezuela, during 2013. The screening included the pulmonary vein FVW assessment by either 2D US or X-flow. Cardiac anomalies were confirmed by late-pregnancy echocardiography, and in three cases fetal autopsies were performed (A. S.-O.).

\section{Case 1}

This was a 40-year-old pregnant woman (3 gravidas, 2 para, 0 abortions [G3, P2, A0]) with a healthy gestation of 13 weeks and 3 days. She had no significant past medical history. The patient was referred to our center for first-trimester screening ultrasound.

Besides increased NT $(6 \mathrm{~mm})$, the rest of the screening markers were within normal limits. However, the cardiac fourchamber view by US B-mode, via transvaginal, suggested the presence of an atrioventricular (AV) canal. The Doppler assessment revealed an A-wave reversal of both DV and pulmonary vein along with a single and central $A V$ canal (-Fig. 2).

Ultimately, the fetal karyotype showed trisomy 21 . The pregnancy was interrupted, but no autopsy was performed since the mother refused.

\section{Case 2}

This was a 35-year-old woman with current gestation of 12 weeks and 6 days. Obstetric history showed G4, P1, A2 (one anembryonic sac, and one trisomy 13). No other pertinent past medical history was reported. Most of the first-trimester markers were within normal limits (-Table $\mathbf{1}$ ). Nevertheless, it was found a marked cephaloabdominal biometry disproportion (cephalic circumference $7.43 \mathrm{~cm}$ with abdominal circumference of $4.99 \mathrm{~cm}$ ) along with a discrete heart asymmetry (smaller left heart), and oligohydramnios (-Fig. 3 ).

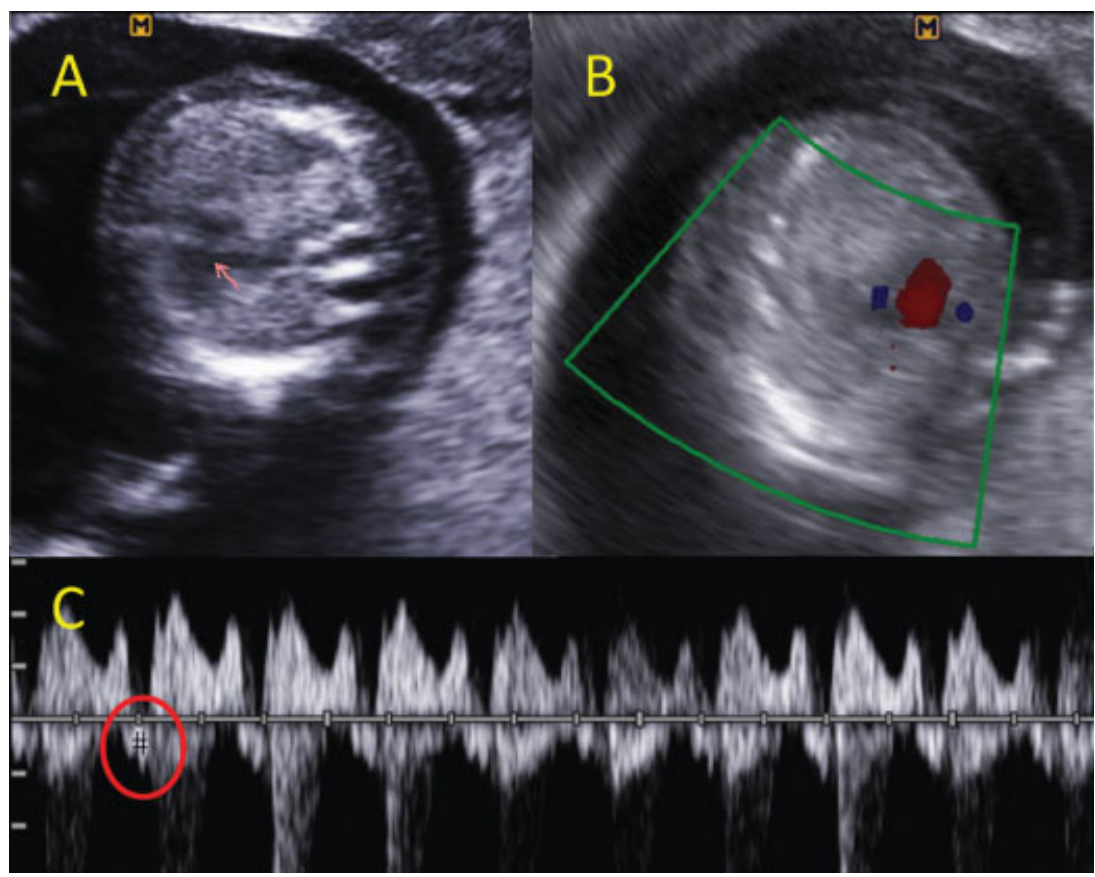

Fig. 2 Atrioventricular canal and pulmonary vein A-wave reversal. (A) B-mode ultrasound showing four-chamber view, arrow points toward atrioventricular canal. (B) Color Doppler shows ventricular inflow through single atrioventricular valve. (C) Circle shows the pulmonary vein A-wave reversal. 
Table 1 First-trimester screening markers by case presented

\begin{tabular}{|c|c|c|c|c|c|c|}
\hline & Case 1 & Case 2 & Case 3 & Case 4 & Case 5 & Case 6 \\
\hline Gestational age & $13 w k+3 d$ & $12 w k+6 d$ & $13 w k+3 d$ & $13 \mathrm{wk}$ & $13 \mathrm{wk}$ & $12 w k+4 d$ \\
\hline Diagnosis & AV canal & $\begin{array}{l}\text { Aortic arch } \\
\text { interruption }\end{array}$ & VSD & $\begin{array}{l}\text { Left ventricle } \\
\text { hypoplasia }\end{array}$ & Functional CM & $\begin{array}{l}\text { Tricuspid } \\
\text { atresia }\end{array}$ \\
\hline \multicolumn{7}{|l|}{ Structural } \\
\hline NT & $6 \mathrm{~mm}$ & Normal & Normal & $6 \mathrm{~mm}$ & $3.2 \mathrm{~mm}$ & Normal \\
\hline NB & + & + & + & + & + & + \\
\hline NPT & Normal & Normal & Normal & Normal & Normal & Normal \\
\hline FMA & Normal & Not rated & Normal & Normal & Normal & Normal \\
\hline IT & Normal & Normal & Normal & Normal & Normal & Normal \\
\hline PCA-CD & Present & Present & Absent & Present & Not rated & Present \\
\hline Heart 4C and OT & $\begin{array}{l}\text { 4C abnormal } \\
\text { (TrV) }\end{array}$ & 4C abnormal (TrV) & $\begin{array}{l}\text { Unable to } \\
\text { obtain }\end{array}$ & 4C abnormal & 4C abnormal & Normal \\
\hline \multicolumn{7}{|l|}{$\begin{array}{l}\text { Doppler (spectral } \\
\text { and/or color) }\end{array}$} \\
\hline DV & Reversal & Reversal & Normal & Reversal & Normal & Normal \\
\hline TV inflow & Normal & Normal & Normal & Regurgitation & Normal & Normal \\
\hline MV inflow & Normal & Normal & Normal & NA & NA & Normal \\
\hline PV & Reversal & Reversal & Reversal & Reversal & Reversal & Normal \\
\hline UA & Normal & Normal & Normal & NA & NA & Normal \\
\hline UTA & Normal & Normal & Normal & Normal & Normal & Normal \\
\hline Karyotype & $47(21) X Y$ & $47, X X Y$ & Refused & $46, X X$ & $46, X Y$ & $46, X Y$ \\
\hline
\end{tabular}

Abbreviations: DV, ductus venosus; FMA, frontomaxillary angle; Heart 4C y OT, 4 chamber and outflow tract views; MT, mitral transvalvular; NA, not available; NB, nasal bone; NPT, nasopalatine triangle; NT, nuchal translucency; PCA-CD, pericallosal artery-color Doppler; PV, pulmonary vein; IT, intracranial translucency; TrV, transvaginal; TV, tricuspid transvalvular; UA, umbilical artery; UTA, uterine artery.

The Doppler examination showed normal AV flow velocity waveforms. However, the DV showed a minimal A-wave reversal along with a frank end-diastolic flow reversal of the pulmonary vein ( - Fig. 4).

Subsequently, karyotype showed a triploidy 47-XXY. Pregnancy was interrupted and followed by anatomopathology. The fetal autopsy demonstrated a complex cardiac malformation described as type B interruption of aortic arch (-Fig. 5).

\section{Case 3}

This was a 41-year-old woman (G3, P2, A0) with a noncomplicated 13 weeks' and 3 days' gestation. Her past medical
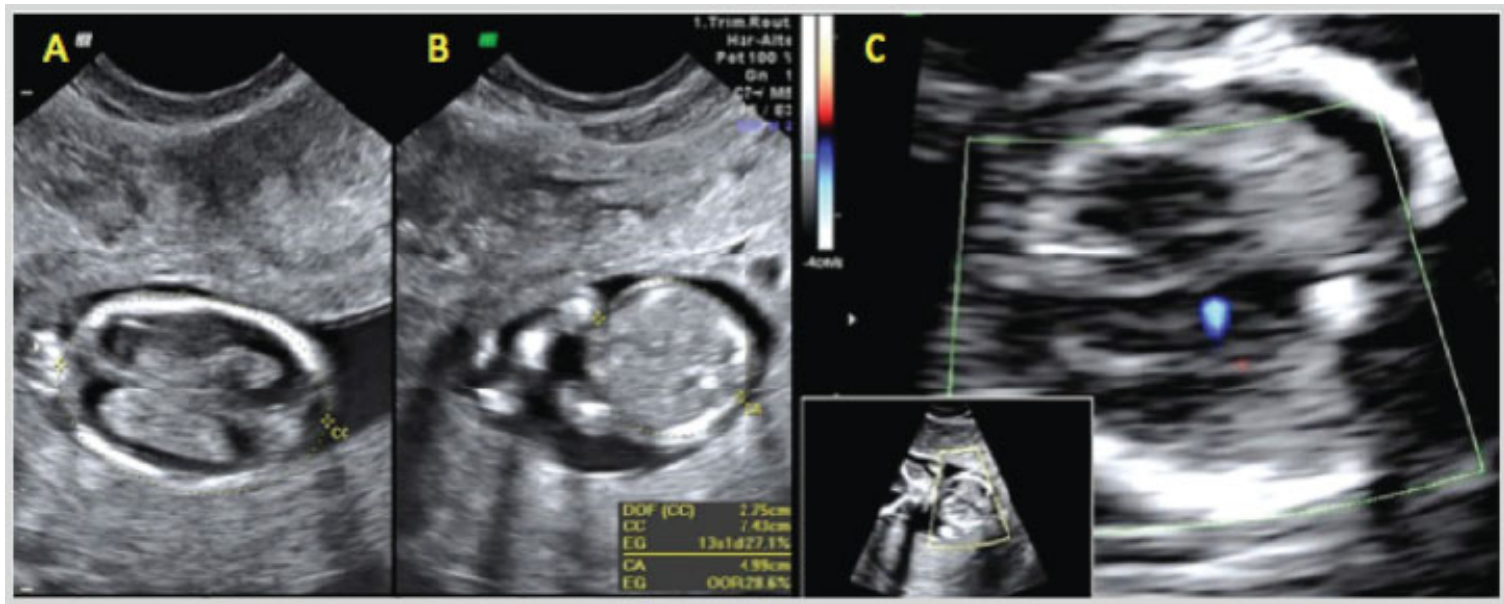

Fig. 3 Severe biometric disproportion and heart asymmetry. (A) Cephalic circumference (CC) $7.43 \mathrm{~cm}$. (B) Abdominal circumference (CA) $4.99 \mathrm{~cm}$. (C) Discrete cardiac asymmetry due to enlarged left-sided chambers. 


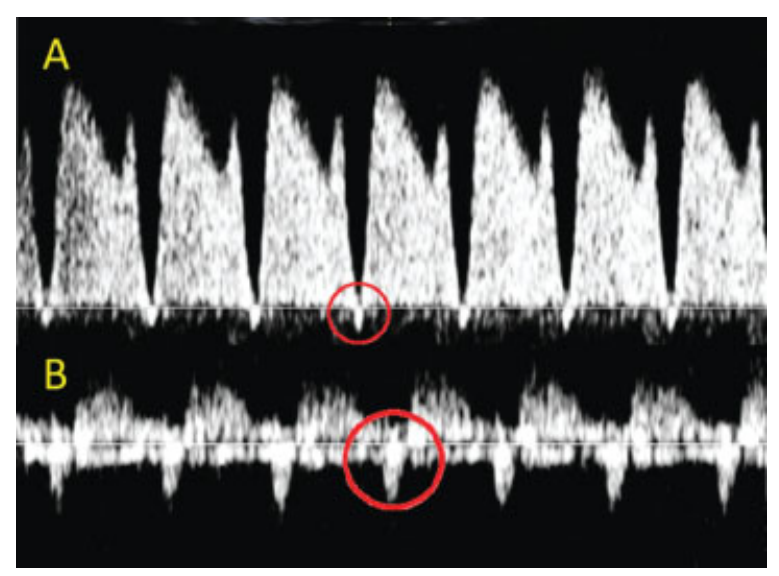

Fig. 4 Doppler assessment of pulmonary vein and ductus venosus flow velocity waveforms. (A) Minimal ductus venosus A-wave reversal. (B) Frank pulmonary vein A-wave reversal.

history was pertinent for prior pregnancy complicated by fetal congenital cardiomyopathy. Most of first-trimester markers were normal ( - Table 1 ). Structural evaluation of the heart, by four-chamber and outflow tract views, could not be obtained. However, Doppler evaluation demonstrated a reversal of pulmonary vein A-wave, with normal DV ( - Fig. 6). Patient refused karyotyping.

Late pregnancy (28 weeks) obstetric ultrasound reported agenesis of cerebellar vermix and intrauterine growth restriction. Gestation was interrupted and fetus was delivered via C-section at 33 weeks due to maternal gestational hypertension complicated by placental insufficiency and oligohydramnios. The newborn weighted $1,300 \mathrm{~g}$ at birth and had Apgar scores of 7 and 8 at 1 and 5 minutes, respectively, but died soon after, secondary to disseminated intravascular coagulation, secondary to fetal hypoxia. Fetal autopsy incidentally demonstrated a major congenital malformation with cyanotic cardiomyopathy and wide ventricular septal defect (VSD).

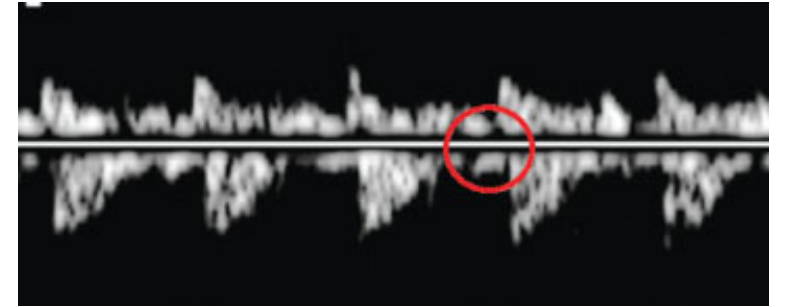

Fig. 6 Doppler assessment of pulmonary vein flow velocity waveforms (FVWs). (A) The circle shows the reversal of pulmonary vein A-wave.

\section{Case 4}

This was a 36-year-old pregnant woman (G1, P0, A0) with a 13 weeks' gestation. She had no significant past medical history. Initial ultrasound reported presence of cystic hygroma and generalized subcutaneous edema. Fetal screening showed abnormal NT (6 mm), but normal nasal bones, nasopalatine triangle, mandibular symphysis, and frontomaxillary angle with normal color Doppler of pericallosal artery ( - Table 1). Cardiac evaluation using a four-chamber view showed a hypoplastic left ventricle (left ventricle $0.1 \mathrm{~cm}$, right ventricle $0.28 \mathrm{~cm}$ ) (-Fig. 7).

The Doppler evaluation demonstrated tricuspid regurgitation and profound end-diastolic flow reversal of pulmonary vein and DV (- Fig. 8). A chorionic villus sampling showed a normal karyotype. Prenatal echography at 18 weeks reported hydropic fetus without cardiac activity. Fetal autopsy revealed a left-sided heart hypoplasia (-Fig. 9).

\section{Case 5}

This was a 22-years-old primigravida (G1, P0, A0) with pregnancy of 13 weeks who presented for routine first-trimester screening. There was no significant past medical history. Firsttrimester screening reported an NT of $3.2 \mathrm{~mm}$, along with hydrops fetalis (pericorporal edema, pericardial effusion, hydrothorax, and ascites). However, remaining markers were within

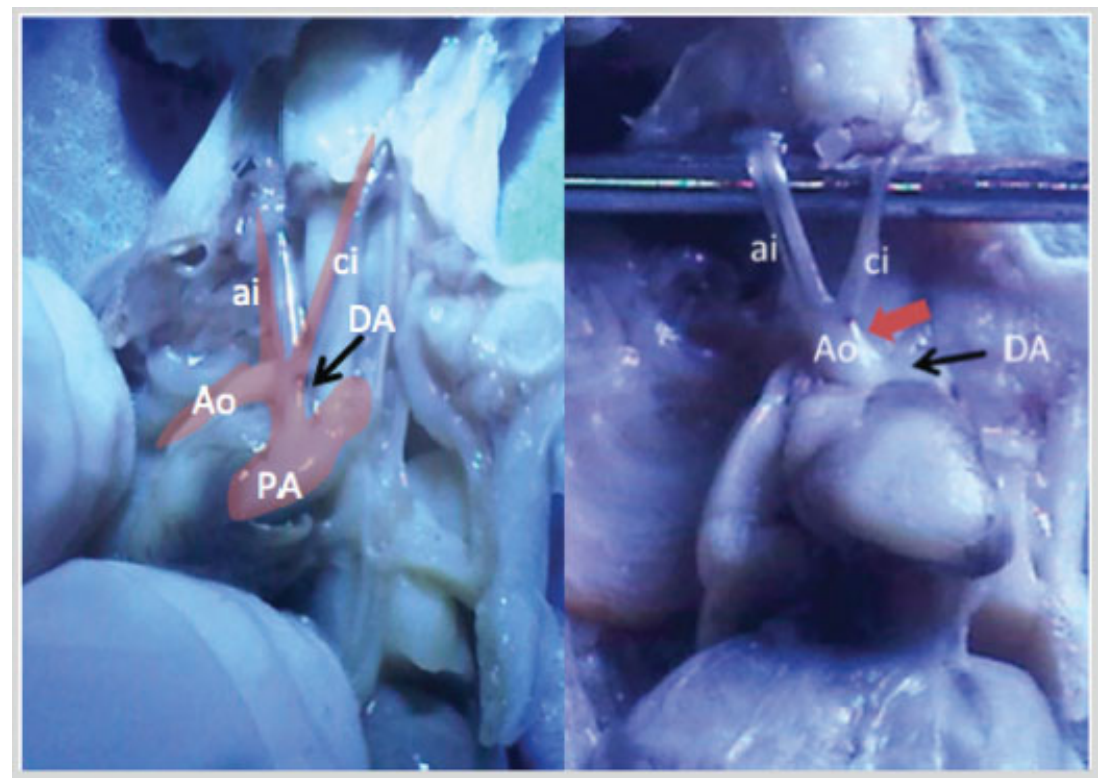

Fig. 5 Fetal autopsy showing type B aortic arch interruption. ai, innominate artery; Ao, aorta; ci, internal carotid artery; DA, ductus arteriosus; PA, pulmonary artery. 


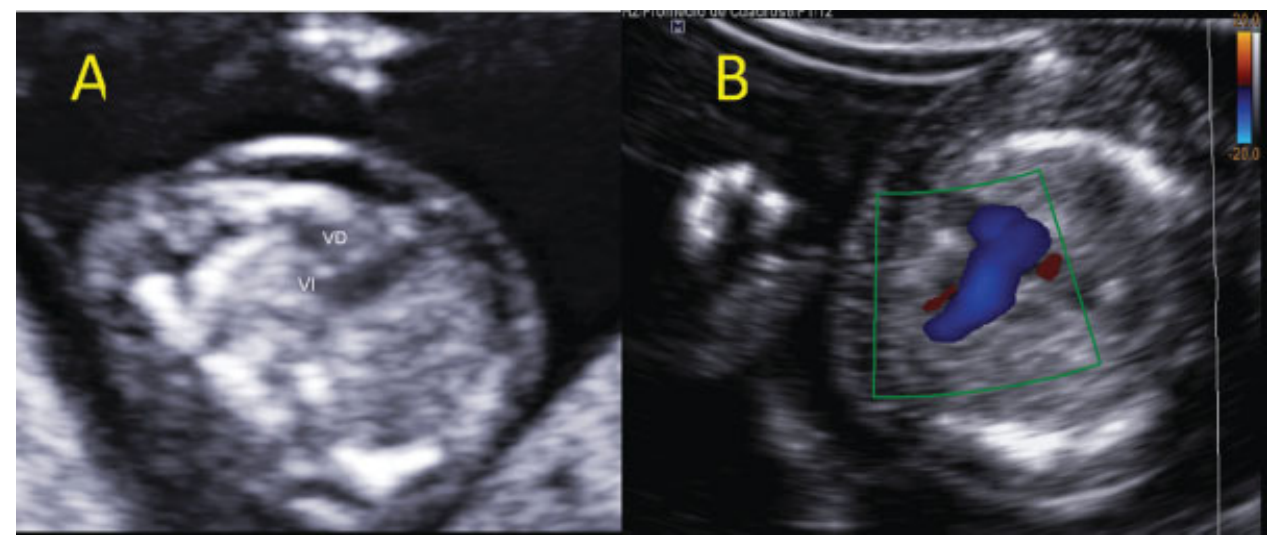

Fig. 7 Assessment of cardiac structure by B-mode US, and color Doppler assessment of atrioventricular inflows. (A) Cardiac four-chamber view showing ventricular asymmetry: Right ventricle (VD) $0.28 \mathrm{~cm}$; left ventricle (VI) $0.10 \mathrm{~cm}$. (B) Doppler color, four-chamber view showing single chamber filling during diastole (right-sided heart).

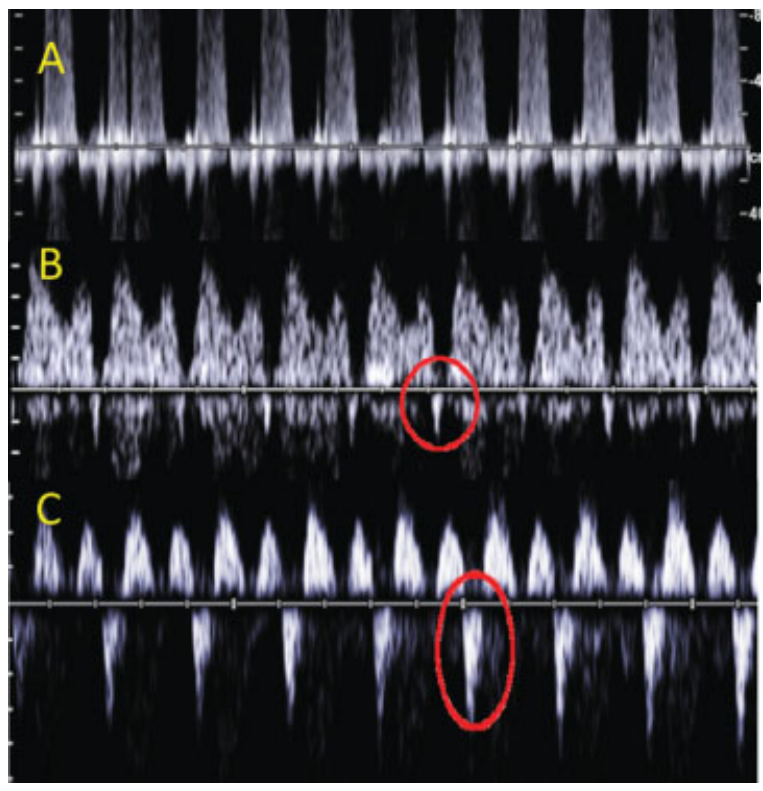

Fig. 8 Doppler assessment tricuspid inflow, ductus venosus, and pulmonary vein flow velocity waveforms (FVWs). (A) Significant tricuspid regurgitation. (B) A-wave reversal of ductus venosus. (C) A-wave reversal of pulmonary vein. normal limits (- Table 1). Cardiac structural assessment was noncontributory. The Doppler evaluation demonstrated isolated A-wave reversal of pulmonary vein (-Fig. 10).

Gestation was interrupted and fetal autopsy was performed. Anatomopathologic evaluation revealed hydrops fetalis, with significant cardiomegaly but otherwise sparred cardiac structure. This findings suggested severe functional cardiomyopathy.

\section{Case 6}

This was a 33-years-old secundigravida (G2, P1, A0) with 12 weeks and 4 days of gestation. There was no significant past medical history. First-trimester markers were within normal limits. Limited structural assessment of the heart using a four-chamber view was normal. The Doppler evaluation demonstrated normal DV, mitral inflow, and even pulmonary vein FVWs (-Table 1). Tricuspid inflow assessment was suspicious but limited due to fetal position (-Fig. 11).

Surprisingly, echocardiographic at 18 weeks showed asymmetry of cardiac chambers due to right ventricle hypoplasia with wide foramen ovale. Outflow tracts were reported normal. Karyotype was normal (46-XY). Subsequent specialized postnatal echocardiography confirmed the right ventricle hypoplasia with type 1 tricuspid atresia and persistent ductus arteriosus (-Fig. 12). The neonate underwent surgical intervention with good outcome. This case was the only one presenting with

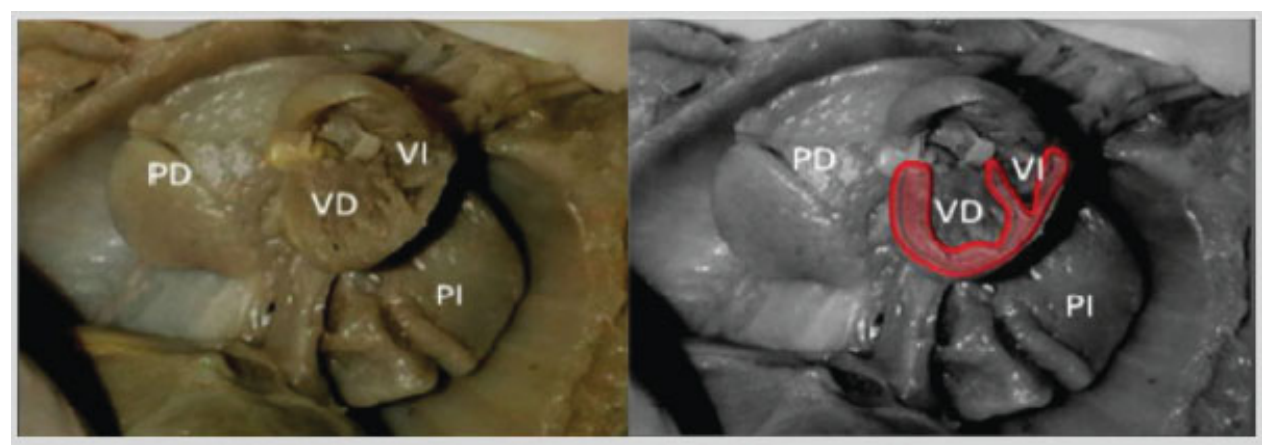

Fig. 9 Fetal autopsy demonstrating the hypoplastic left ventricle. PD, right lung; PI, left lung; VD, right ventricle; VI, left ventricle. 


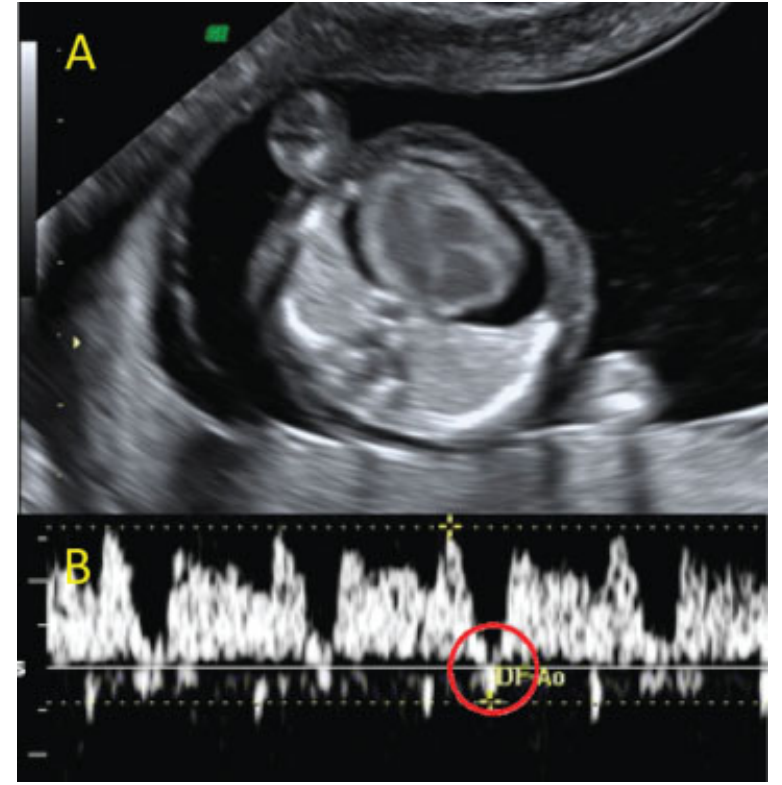

Fig. 10 Structural assessment of the fetus by B-mode US and Doppler evaluation of pulmonary vein flow velocity waveforms (FVWs). (A) Fetal cross-sectional plane showing hydrothorax, pericardial effusion, cardiomegaly, and pericorporal edema. (B) Circle shows pulmonary vein A-wave reversal.

normal pulmonary vein FVW despite the presence of CHD. The absence of pulmonary vein A-wave reversal could be explained by the predominant right-sided cardiac involvement.

\section{Discussion}

The evaluation of pulmonary vein FVW has been a focus of attention in recent decades to improve detection rate of CHD. To our knowledge, this is the first series reporting pulmonary vein end-diastolic reversal as a CHD screening add-on during early pregnancy. It is known that an increased NT ( $>95$ th percentile) along with reversal of DV could detect $47.1 \%$ of heart defects. ${ }^{11}$ However, when NT is normal, DV would only pick approximately $20 \%$ of the instances of CHD missed by NT. ${ }^{11}$ The current series

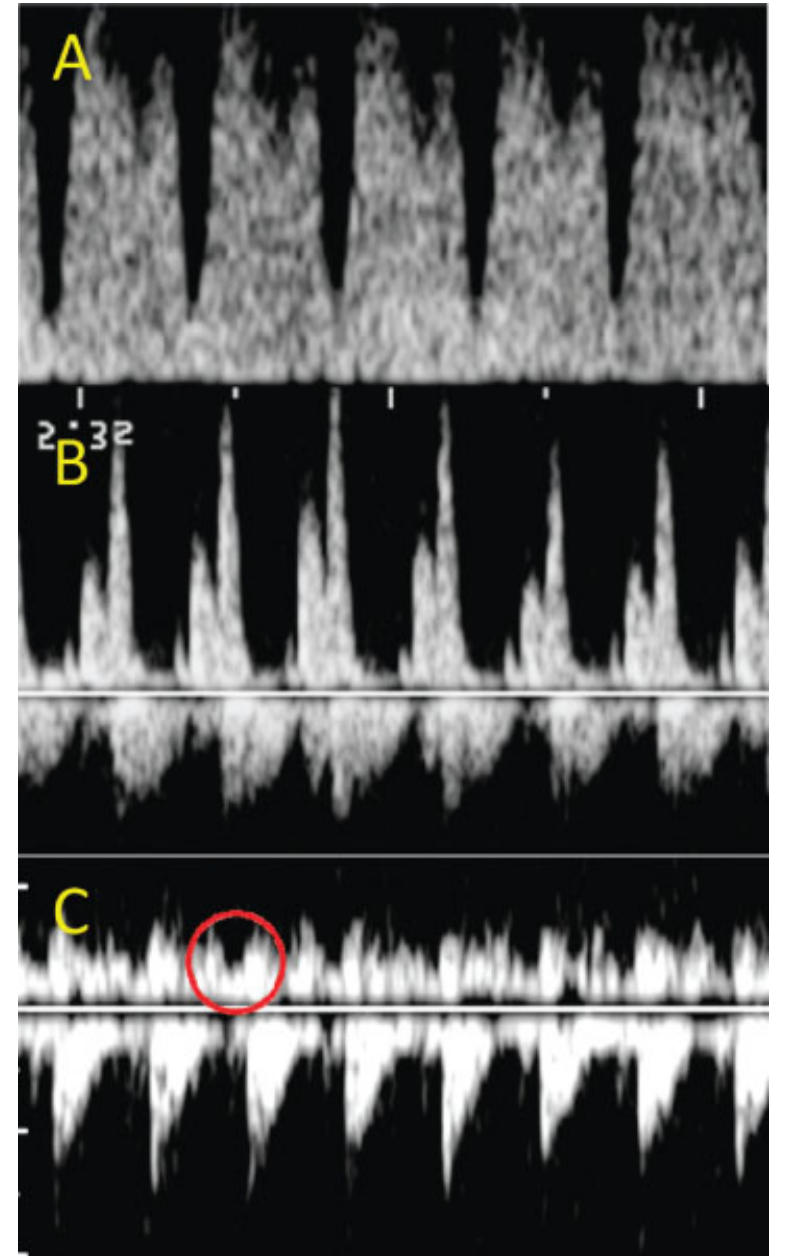

Fig. 11 Atrioventricular inflow, ductus venosus, and pulmonary vein flow velocity waves (FVWs) assessment by Doppler. (A) Forward flow during ductus venosus A-wave. (B) Normal mitral inflow. (C) Normal pulmonary vein end-diastolic forward flow.

mirrors these findings. For instance, the DV and NT predicted $50 \%$ of the CHD, whereas isolated DV reversal only identified $16.6 \%$ of the cases. Similarly, the combination of markers (increased NT, tricuspid insufficiency, and reversal of DV) yields a rate of CHD detection around $57.6 \%{ }^{12}$ In our case series, the

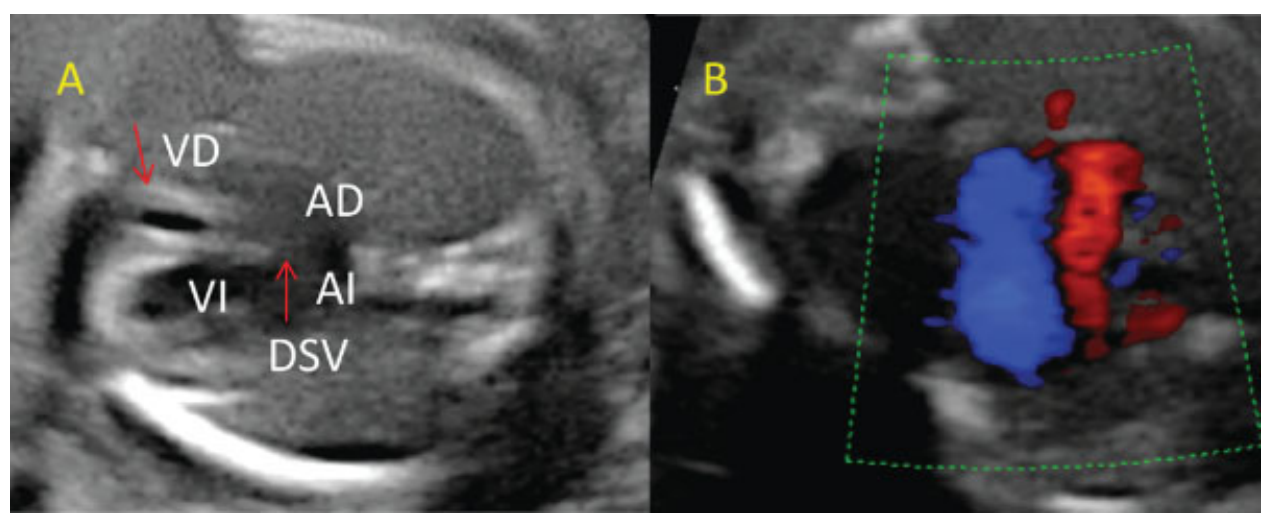

Fig. 12 Ultrasound by B-mode and color Doppler demonstrating the right ventricular hypoplasia with tricuspid atresia. (A) Ultrasound B-mode showing hypoplastic right ventricle. AD, right atrium; AI, left atrium; DSV, ventricular septal defect; VD, right ventricle; VD, right ventricle; VI, left ventricle. (B) Tricuspid atresia, in the setting of retrograde filling of right ventricle by color Doppler. 
combination of markers identified $66.6 \%$ of $\mathrm{CHD}$ cases. More importantly, when pulmonary vein assessment was included, the rate of detection rose to $83.3 \%$. Hence, more studies are needed to prove these findings.

The presence of A-wave reversal in our case with hypoplastic left ventricle is supported by the high rate of reversal among cases with left atrial obstruction in the Lenz study. ${ }^{13}$ The reversal can be explained by the increase in the intra-atrial pressure due to atrial outflow obstruction. On the other hand, Lenz et al reported pulmonary vein reversal in only one of eight cases with left ventricular outflow tract obstruction. ${ }^{13}$ Our case with aortic arch interruption resembles the single case with A-wave reversal in the Lenz study; that fetus had severe aortic arch hypoplasia. It could be that left ventricular outflow obstructions have more compensation capacity than atrial anomalies; therefore it requires a more severe form to cause a reversal of pulmonary vein end-diastolic flow. Similarly, our case with right ventricular hypoplasia mirrored findings of the Lenz study ${ }^{13}$ cases with predominant right-sided cardiac involvement reporting normal pulmonary vein FVW.

Contrary to Lenz ${ }^{13}$ findings, our cases with AV canal and VSD showed reversal of pulmonary vein end-diastolic flow. These contrasting findings might represent different degrees of left-ventricular hemodynamic compromise. This statement can be supported by our case with pure functional cardiomyopathy. In this case, the pulmonary vein A-wave reversal is explained by solely left ventricular hemodynamic compromise in a failing heart.

In our case series, all those cardiac anomalies with predominant left ventricular involvement had pulmonary vein A-wave reversal, whereas the case of isolated right ventricular compromise demonstrated a normal end-diastolic waveform. It could be hypothesized that Doppler examination of the pulmonary vein could function as a specific visor for left-sided heart anomalies. ${ }^{4}$

We understand the significant limitations of a case series, including the very small sample, which prevents the formulation of definite conclusions. Therefore, further studies are needed to test the proposed theories.

\section{Conclusion}

The addition of pulmonary vein FVW assessment to the current CHD screening bundle could increase the rate detection of cardiac anomalies. This pilot study suggests that pulmonary vein end-diastolic flow reversal favors detection of left-sided CHD over the right-sided ones.

\section{References}

1 Pereira S, Ganapathy R, Syngelaki A, Maiz N, Nicolaides KH. Contribution of fetal tricuspid regurgitation in first-trimester screening for major cardiac defects. Obstet Gynecol 2011; 117(6):1384-1391

2 Carvalho JS, Allan LD, Chaoui R, et al; International Society of Ultrasound in Obstetrics and Gynecology. ISUOG Practice Guidelines (updated): sonographic screening examination of the fetal heart. Ultrasound Obstet Gynecol 2013;41(3):348-359

3 DeVore GR. Assessment of venous flow in normal and high risk fetuses. Is the future now? Prenat Neonatal Med 1996;1:329-342

4 Kiserud T. Fetal venous circulation-an update on hemodynamics. J Perinat Med 2000;28(2):90-96

5 Moon-Grady A, Shahanavaz S, Brook M, Rodriguez H, Hornberger LK. Can a complete fetal echocardiogram be performed at 12 to 16 weeks' gestation? J Am Soc Echocardiogr 2012;25(12): 1342-1352

6 Dong FQ Zhang YH, Li ZA, Hou ZZ, He XJ, Guo YZ. Evaluation of normal fetal pulmonary veins from the early second trimester by enhanced-flow (e-flow) echocardiography. Ultrasound Obstet Gynecol 2011;38(6):652-657

7 Zhang Y, Ding C, Fan M, et al. Evaluation of normal fetal pulmonary veins using B-flow imaging with spatiotemporal image correlation and by traditional color Doppler echocardiography. Prenat Diagn 2012;32(12):1186-1191

8 Schenone AL, Giugni G, Schenone MH, Majdalany D. A new cardiac marker during first trimester screening? Detection of fetal pulmonary veins by Doppler B mode and X flow between 12-15 weeks of gestation. Cardiology 2014;128(Suppl 1):222

9 Lenz F, Chaoui R. Reference ranges for Doppler-assessed pulmonary venous blood flow velocities and pulsatility indices in normal human fetuses. Prenat Diagn 2002;22(9):786-791

10 Zhu W, Yang XH, Wang XY. Evaluation of hemodynamic changes of finger tip capillary vessels in patients with primary hypertension using E-Flow imaging technology. Chinese J Ultrasonographic Image 2008;17:504-507

11 Chelemen T, Syngelaki A, Maiz N, Allan L, Nicolaides KH. Contribution of ductus venosus Doppler in first-trimester screening for major cardiac defects. Fetal Diagn Ther 2011; 29(2):127-134

12 Papatheodorou SI, Evangelou E, Makrydimas G, Ioannidis JP. Firsttrimester ductus venosus screening for cardiac defects: a metaanalysis. BJOG 2011;118(12):1438-1445

13 Lenz F, Chaoui R. Changes in pulmonary venous Doppler parameters in fetal cardiac defects. Ultrasound Obstet Gynecol 2006; 28(1):63-70 